University of New Hampshire

University of New Hampshire Scholars' Repository

Space Science Center

Institute for the Study of Earth, Oceans, and

Space (EOS)

1996

\title{
The angular distribution of COMPTEL Gamma-Ray bursts
}

\author{
R M. Kippen \\ University of New Hampshire - Main Campus \\ James M. Ryan \\ University of New Hampshire, James.Ryan@unh.edu \\ A Connors \\ University of New Hampshire - Main Campus \\ Mark L. McConnell \\ University of New Hampshire - Main Campus, mark.mcconnell@unh.edu \\ H Hartmann \\ Clemson University
}

See next page for additional authors

Follow this and additional works at: https://scholars.unh.edu/ssc

Part of the Astrophysics and Astronomy Commons

\section{Recommended Citation}

The angular distribution of COMPTEL Gamma-Ray bursts Kippen, R. M. and Ryan, J. and Connors, A. and McConnell, M. and Hartmann, D. H. and Winkler, C. and Hanlon, L. O. and Schönfelder, V. and Greiner, J. and Varendorff, M. and Collmar, W. and Hermsen, W. and Kuiper, L., AIP Conference Proceedings, 384, 436-440 (1996), DOI:http://dx.doi.org/10.1063/1.51697

This Conference Proceeding is brought to you for free and open access by the Institute for the Study of Earth, Oceans, and Space (EOS) at University of New Hampshire Scholars' Repository. It has been accepted for inclusion in Space Science Center by an authorized administrator of University of New Hampshire Scholars' Repository. For more information, please contact Scholarly.Communication@unh.edu. 


\section{Authors}

R M. Kippen, James M. Ryan, A Connors, Mark L. McConnell, H Hartmann, C Winkler, L O. Hanlon, V Schonfelder, J Greiner, M Varendorff, W Collmar, W Hermsen, and L Kuiper 


\section{AIP $\mid$ Proceedings}

\section{The angular distribution of COMPTEL Gamma-Ray bursts}

R. M. Kippen, J. Ryan, A. Connors, M. McConnell, D. H. Hartmann, C. Winkler, L. O. Hanlon, V. Schönfelder, J. Greiner, M. Varendorff, W. Collmar, W. Hermsen, and L. Kuiper

Citation: AIP Conference Proceedings 384, 436 (1996); doi: 10.1063/1.51697

View online: http://dx.doi.org/10.1063/1.51697

View Table of Contents:

http://scitation.aip.org/content/aip/proceeding/aipcp/384?ver=pdfcov

Published by the AIP Publishing

\section{Articles you may be interested in}

New Statistical Results on the Angular Distribution of GammaRay Bursts AIP Conf. Proc. 1000, 52 (2008); 10.1063/1.2943523

The Results of Statistical Tests of the Angular Distribution of GammaRay Bursts AIP Conf. Proc. 662, 163 (2003); 10.1063/1.1579329

Testing the intrinsic randomness in the angular distributions of gamma-ray bursts AIP Conf. Proc. 526, 102 (2000); 10.1063/1.1361516

COMPTEL observations of cosmic gammaray bursts AIP Conf. Proc. 265, 22 (1991); 10.1063/1.42837

COMPTEL images locations of gammaray bursts AIP Conf. Proc. 265, 77 (1991); 10.1063/1.42782 


\title{
The Angular Distribution of COMPTEL Gamma-Ray Bursts
}

\author{
R.M. Kippen*, J. Ryan*, A. Connors*, M. McConnell*, \\ D.H. Hartmann ${ }^{\ddagger}$, C. Winkler ${ }^{\ddagger}$, L.O. Hanlon ${ }^{\ddagger}$, V. Schönfelder ${ }^{\S}$, \\ J. Greiner ${ }^{\S}$, M. Varendorff ${ }^{\S}$, W. Collmar ${ }^{\S}$, W. Hermsenll \\ and L. Kuiperll
}

* Space Science Center, University of New Hampshire, Durham, NH 03824

${ }^{\dagger}$ Dept. of Physics and Astronomy, Clemson University, Clemson, SC 29634

${ }^{\ddagger}$ Astrophysics Division, ESA/ESTEC, NL-2200 AG Noordwijk, NL

${ }^{\S}$ Max-Planck-Institut für Extraterrestrische Physik, D-85748 Garching, FRG

"SRON-Utrecht, Sorbonnelaan 2, 3584 CA Utrecht, NL

The superior burst location capability of the COMPTEL instrument aboard the Compton Gamma-Ray Observatory allows us to study the small-scale angular distribution of burst sources with good sensitivity even though the number of burst detections is small. We accumulate four years (April 1991 - April 1995) of observations to form a catalog of 27 burst locations whose mean $1 \sigma$ uncertainty is $\sim 1^{\circ}$. We find that the COMPTEL bursts are consistent with an isotropic distribution of sources, yet the spatial coincidence of two of the bursts within COMPTEL's angular resolution indicates the possibility of repetition. This possibility is studied using the two-point angular correlation function and the nearest neighbor statistic. Model dependent upper limits on the fraction of repeating sources are derived.

\section{INTRODUCTION}

The isotropic, inhomogeneous distribution of burst sources derived from BATSE data has led to the general acknowledgment that GRBs could be of cosmological origin. If bursts are associated with cosmologically distant galaxies, we do not expect (in most scenarios) to observe multiple events from the same direction. The small-scale angular distribution of burst sources thus plays a vital role in confirming or dispelling the cosmological hypothesis. Numerous investigations using BATSE data have yielded conflicting results due to BATSE's limited angular resolution of a few degrees at best. However, most recent analyses agree that the BATSE burst locations are consistent with isotropy even on small angular scales - indicating that burst recurrence is either rare or non-existent (1-3).

In the present study, we use the burst localization capabilities of The Imaging Compton Telescope (COMPTEL) to independently investigate the angular distribution of GRBs. Although it detects far fewer bursts, COMPTEL

(C) 1996 American Institute of Physics 

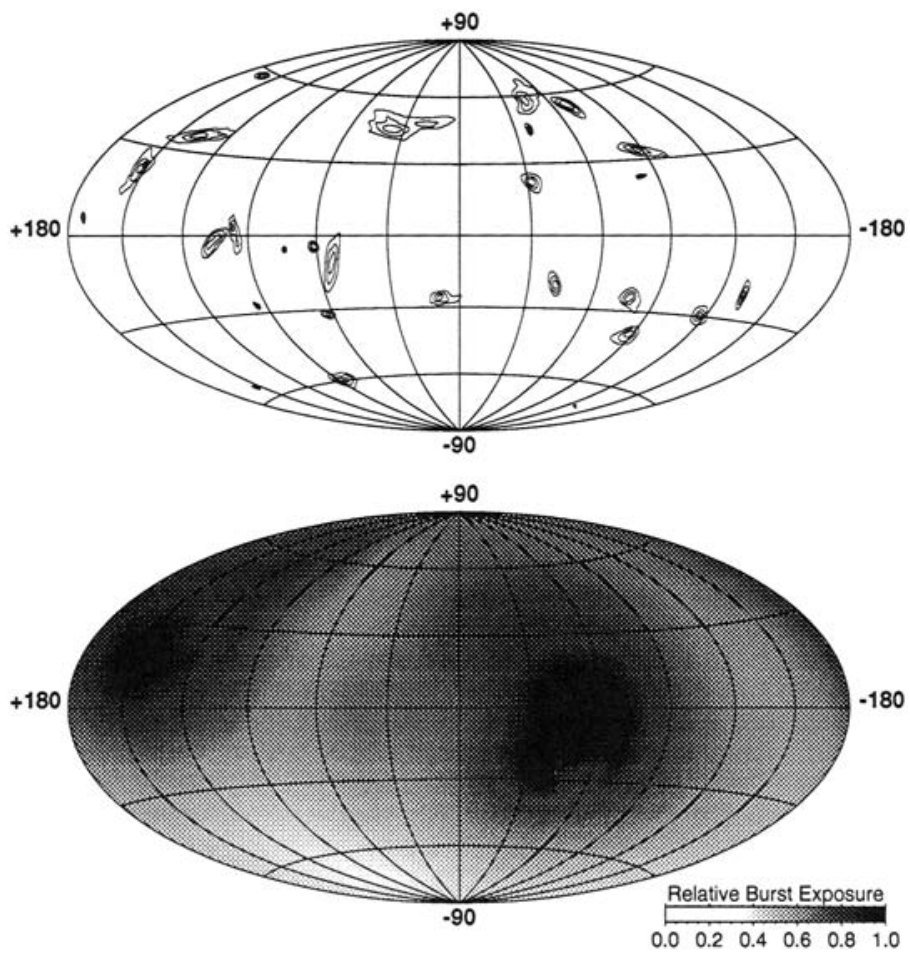

FIG. 1. Statistical location uncertainty contours $(1,2,3 \sigma$ confidence $)$ of 27 bursts imaged by COMPTEL in four years of observations (top) and corresponding "exposure" map (bottom) plotted in Galactic coordinates.

has the advantage over BATSE that its burst localization accuracy is superior $\left(\sim 1^{\circ}\right)$. This makes even a modest sample of COMPTEL burst locations particularly valuable for investigating the small-scale angular distribution of burst sources.

\section{OBSERVATIONS AND BURST LOCALIZATION}

Gamma-ray bursts are observed regularly within the field-of-view ( 1-3 sr depending on fluence) of the main COMPTEL "telescope" operating mode $(\sim 0.75-30 \mathrm{MeV})$. Lacking an on-board transient triggering system, these bursts are identified by searching for excess numbers of telescope "events" (4) at the times of all BATSE burst triggers. This technique is effective since BATSE is a far more sensitive detector at energies where bursts emit most of their power.

In the four year period from April 1991 through April 1995, 27 significant $(Z 4 \sigma)$ detections have been identified out of more than 230 candidate BATSE triggers (5). Each of these bursts has been localized through direct imaging of 


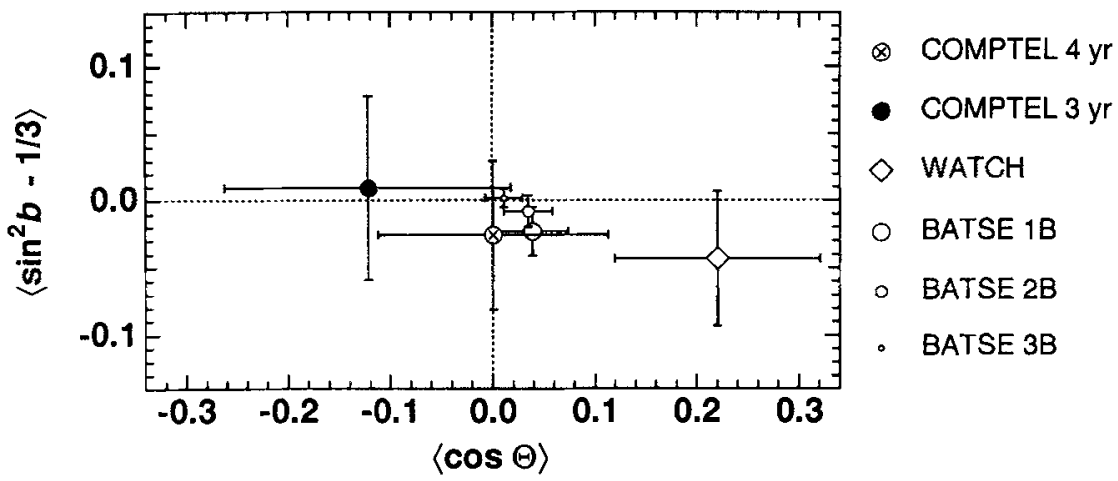

FIG. 2. Galactic dipole and quadrupole moments derived from COMPTEL burst localizations are compared to BATSE (2) and Granat-WATCH (8) results.

the $\mathrm{MeV}$ photon events using a maximum likelihood technique that provides quantitative constraints on the source direction and flux (6). Statistical burst location accuracy ( $1 \sigma$ confidence) ranges from $<0.5^{\circ}$ for the strongest events to $\sim 2^{\circ}$ for the weakest, with a mean of $\sim 1^{\circ}$ for all 27 bursts (see Figure 1). Simulations and comparisons with other instruments indicate that systematic location errors are $<0.5^{\circ}$.

\section{SPATIAL ANALYSIS}

The angular distribution of COMPTEL burst directions is analyzed using two inclusive samples: the 27 bursts detected in four years of observations and the 18 bursts (7) of the first three years. Although the full sky has been observed by COMPTEL, several regions (most notably Virgo and the Galactic center) have received substantially $(\sim 30-50 \%)$ higher exposure. For each sample interval, a map of COMPTEL's non-uniform probability of detecting a burst on the sky (or "exposure") has been computed by accumulating data from the many pointed observation periods (Figure 1).

As shown in Figure 2, Galactic dipole and quadrupole moments corrected for the non-uniform exposure indicate that both the three year and four year burst location samples are consistent (on large scales) with an isotropic angular distribution of sources. Uncertainties in these quantities caused by burst location errors are negligible compared to the large statistical errors due to the small sample size.

Small-scale structure in the angular distribution is examined using the (now standard) two-point angular correlation function $w(\theta)$ and the nearest neighbor statistic $N N(\theta)(1)$. Both of these statistical tools are sensitive to smallscale clustering, which could indicate repeated bursts from individual sources. To account for burst location errors, observed $w(\theta)$ and $N N(\theta)$ distributions are evaluated by averaging over points sampled randomly from the COMP- 

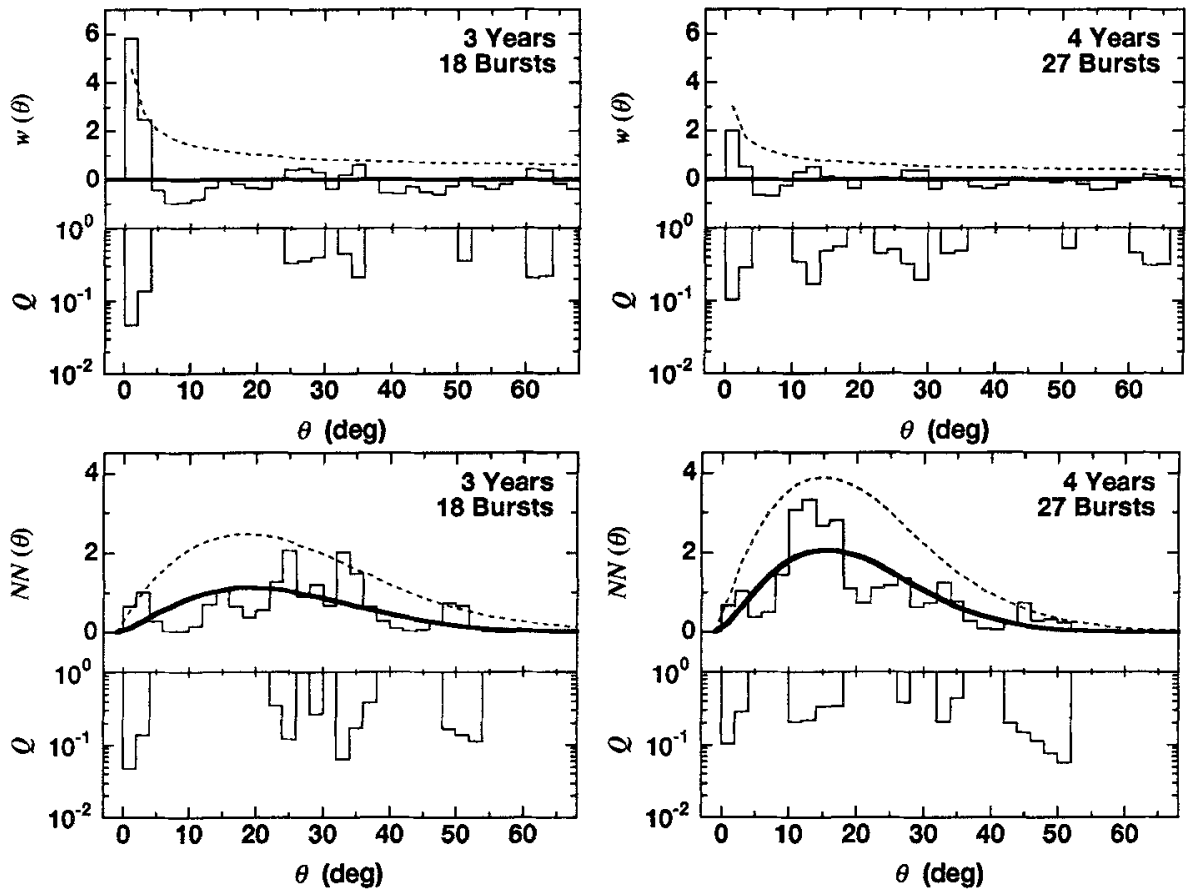

FIG. 3. Angular correlation functions (upper plots) and nearest neighbor distributions (lower plots) of two samples of COMPTEL burst locations. Each plot compares the average observed distribution (solid histogram) with the distributions (solid curve) and $1 \sigma$ statistical deviations (dashed curve) expected from isotropy. The statistical significance $Q$ of observed deviations from isotropy are indicated in the lower panels of each plot.

TEL maximum likelihood burst localizations (i.e., Figure 1). Each observed average distribution is compared to the null hypothesis of isotropy, weighted with COMPTEL's non-uniform sky exposure map, as shown in Figure 3. The significance $Q$ of observed deviations in excess of the isotropic hypothesis is evaluated through Monte Carlo simulation.

In the three year sample of $18 \mathrm{GRBs}$, there is evidence of a marginally significant excess of bursts with small angular separations $\left(\theta \leqslant 2^{\circ}\right)$. The cause of this excess is that two of the COMPTEL bursts (GRB 930704 and GRB 940301) are localized to the same direction within their combined location errors. The probability of this coincidence occurring by chance based on either angular correlation or nearest neighbor analyses is $Q \lessgtr 3 \%$ - suggesting the possibility of burst recurrence (6). However, the small-angle excess is not observed in the full four year burst sample, indicating that no other coincidences have since been detected, thus diluting the significance of the GRB 930704/940301 overlap. 


\section{DISCUSSION}

Given their limited numbers, the COMPTEL burst location samples provide little more than a consistency check on BATSE's unsurpassed measurements of the large-scale angular distribution of burst sources. However, the COMPTEL localizations do provide a valuable, independent measure of the small-scale properties of this distribution by virtue of their superior location accuracy. Results from the 27 burst sample presented here indicate that the COMPTEL burst locations are statistically consistent with an isotropic distribution of sources. If we accept this result, models of burst recurrence can be constrained. As an example, we fit a simple, one parameter $\left(f_{\mathrm{r}}\right.$, the fraction of sources that produce repeated bursts) burst recurrence model (9) to the COMPTEL observations and find that this particular model can be rejected with $95 \%$ confidence when $f_{r}>23 \%$. Similar analysis of the first 260 BATSE bursts was not as constraining due to BATSE's poor location accuracy (9). Unfortunately, models predicting fewer bursts per repeating source are not constrained by COMPTEL observations because we would not yet expect to see many recurrences in such small samples.

The preceding arguments still do not rule out the possibility that the coincident bursts GRB 930704 and GRB 940301 were produced by a single source. Nor can this possibility be excluded with the combined observations of COMPTEL, BATSE, EGRET and the Interplanetary Network. We can, however, conclude that if recurrence exists, it must be a relatively rare phenomenon. Continued observations may yet yield a more definitive answer.

Acknowledgements. This research was supported by the Compton Observatory Guest Investigator Program under NASA contract NAG5-2350. The COMPTEL project is supported by NASA under contract NAS5-26645, by the German government through DARA grant 50 QV 90968 and by the Netherlands Organization for Scientific Research (NWO). JG acknowledges the support of DARA under contract FKZ 50 OR 9201.

\section{REFERENCES}

1. C.A. Meegan et al., ApJ 446, L15 (1995).

2. C.A. Meegan et al., ApJ in press.

3. M. Tegmark et al., ApJ in press.

4. V. Schönfelder et al., ApJS 86, 657 (1993).

5. R.M. Kippen et al., ApJ in preparation.

6. R.M. Kippen et al., A\&A 293, L5 (1995).

7. R.M. Kippen et al., ApSS 231, 231 (1995).

8. A.J. Castro-Tirado et al., in Gamma-Ray Bursts, ed. G.J. Fishman, J.J. Brainerd \& K. Hurley (AIP 307, New York, 1994), p. 17.

9. T.E. Strohmayer, E.E. Fenimore \& J.A. Miralles, ApJ 432, 665 (1994). 